\title{
Distinct tissue distributions and subcellular localizations of differently phosphorylated forms of the myosin regulatory light chain in Drosophila
}

\author{
Liang Zhang and Robert E. Ward IV 1 \\ Department of Molecular Biosciences, University of Kansas, Lawrence, Kansas 66045
}

\begin{abstract}
Nonmuscle myosin II (myosin hereafter) has well-established roles in generating contractile force on actin filaments during morphogenetic processes in all metazoans. Myosin activation is regulated by phosphorylation of the myosin regulatory light chain (MRCL, encoded by spaghetti squash or sqh in Drosophila) first on Ser21 and subsequently on Thr20. These phosphorylation events are positively controlled by a variety of kinases including myosin light chain kinase, Rho kinase, citron kinase, and AMP kinase and are negatively regulated by myosin phosphatase. The activation of myosin is thus highly regulated and likely developmentally controlled. In order to monitor the activity of myosin during development, we have generated antibodies against the monophosphorylated (Sqh1P) and diphosphorylated (Sqh2P) forms of Sqh. We first show that the antibodies are highly specific. We then used these antibodies to monitor myosin activation in wild type Drosophila tissues. Interestingly, Sqh1P and Sqh2P show distinct patterns of expression in embryos. Sqh1P is expressed nearly ubiquitously and outlines cells consistent with a junctional localization, whereas Sqh2P is strongly expressed on the apical surfaces and in filopodia of tissues undergoing extensive cell shape change or cell movements including the invaginating fore- and hindgut, the invaginating tracheal system, the dorsal pouch and the dorsal most row of epidermal (DME) cells during dorsal closure. In imaginal discs, Sqh1P predominantly localizes in the adherens junction, whereas Sqh2P locates to the apical domain. These antibodies thus have the potential to be very useful in monitoring myosin activation for functional studies of morphogenesis in Drosophila.
\end{abstract}

\section{Keywords \\ Drosophila; myosin; myosin regulatory light chain; spaghetti squash; dorsal closure; morphogenesis}

\section{Results and Discussion}

Nonmuscle myosin II (referred to as myosin hereafter) is a motor protein that binds reversibly to actin filaments and generates contractile forces necessary for a variety of

(C) 2010 Elsevier B.V. All rights reserved.

${ }^{3}$ Corresponding author: Robert E. Ward IV, Department of Molecular Biosciences, University of Kansas, 1200 Sunnyside Ave., Lawrence, Kansas 66045, robward@ ku.edu, Phone: (785) 864-5235, Fax: (785) 864-5294.

Publisher's Disclaimer: This is a PDF file of an unedited manuscript that has been accepted for publication. As a service to our customers we are providing this early version of the manuscript. The manuscript will undergo copyediting, typesetting, and review of the resulting proof before it is published in its final citable form. Please note that during the production process errors may be discovered which could affect the content, and all legal disclaimers that apply to the journal pertain. 
cellular processes including cell shape changes, cell movements, cytokinesis, maintenance of cell morphology and polarity, and intracellular vesicle transport (reviewed in Conti and Adelstein, 2008; Vicente-Manzanares et al., 2009). Consistent with these roles, loss-offunction mutations in myosin in various organisms result in severe developmental defects and are inevitably lethal. For example, in Dictyostelium, cells that lack either myosin heavy chain or the regulatory light chain undergo cytokinesis with greatly decreased efficiency, have dramatic reduction of cell polarity, show greatly reduced motility, cease development after aggregation and fail to form fruiting bodies (reviewed in Bosgraaf and van Haastert, 2006). In Drosophila, strong loss-of-function mutations in the myosin heavy chain (encoded by zipper or zip) are embryonic lethal with defects in axon pathfinding, dorsal closure and head involution (Young et al., 1993). Hypomorphic zip alleles produce malformed adult legs due to defects in cell shape changes during imaginal disc morphogenesis (Gotwals and Fristrom, 1991; Halsell and Kiehart, 1998). Similarly, mutations in the myosin regulatory light chain (encoded by spaghetti squash or sqh) result in disrupted cytokinesis, defects in imaginal disc morphogenesis including eversion of the legs and wings, eye morphogenesis, and oogenesis (Edwards and Kiehart, 1996; Karess et al., 1991).

Myosin molecules are hexamers composed of a pair of heavy chains (MHC), a pair of essential light chains, and a pair of regulatory light chains (MRLC). The activity of myosin is mainly controlled by phosphorylation and dephosphorylation of MRLC (reviewed in Tan et al., 1992). Phosphorylation of Ser19 on MRLC (which corresponds to Ser21 in Drosophila Sqh) can greatly increase the actin-dependent myosin ATPase activity and thus the motor activity of myosin (Ikebe et al., 1988). Subsequent phosphorylation of Thr18 (Thr20 in Sqh) can further increase myosin activity (Ikebe and Hartshorne, 1985; Ikebe et al., 1986; Ikebe et al., 1988). Protein kinases including myosin light chain kinase (MLCK), Rho-associated protein kinase (ROK), Rho-dependent Citron kinase, AMP-activated kinase, leucine zipper interacting kinase (ZIPK) and myotonic dystrophy kinase-related CDC42binding kinase can phosphorylate and activate MRLC (Kosako et al., 2000; Lee et al., 2007; Tan et al., 1992; Ueda et al., 2002; Vicente-Manzanares et al., 2009; Yamashiro et al., 2003). Myosin phosphatase acts antagonistically, dephosphorylating MRLC, thereby leading to inactivation of myosin (Hartshorne, 1998; Vereshchagina et al., 2004).

\subsection{Antibodies specific for phosphorylated forms of Sqh}

To identify spatial and temporal patterns of myosin activation during Drosophila development, we generated two site-specific antibodies against the phosphorylated forms of Sqh: one directed against the monophosphorylated form (with phospho-Ser21; referred to hereafter as Sqh1P), and the other directed against the diphosphorylated form (with phospho-Thr20 and phospho-Ser21; referred to as Sqh2P). We also generated an antibody against full-length Sqh protein to use as a control (initially described in Wang and Ward, 2010). To test the specificity of these antibodies, we conducted western blotting of lysates from wild type imaginal discs. All three antibodies recognized a single band of $\sim 21-\mathrm{kD}$ on these blots, suggesting that all three antibodies recognize endogenous Sqh in this tissue (Fig. 1A). To confirm this notion, we used the antibodies on blots containing protein samples extracted from $y w c v s q h^{A x 3} ; P\{s q h-G F P\}$ animals. $s q h^{A x 3}$ is an amorphic allele that produces no Sqh protein (Jordan and Karess, 1997), whereas sqh-GFP encodes a full-length Sqh protein tagged with Green Fluorescent Protein (GFP). The recombinant protein has a predicted molecular mass of $\sim 48-\mathrm{kD}$. All three antibodies recognize only a single band of $\sim 48$-kD from these lysates, consistent with the antibodies specifically recognizing the recombinant Sqh-GFP (Fig. 1A).

We next performed Urea-glycerol-PAGE followed by immunoblotting to further test the specificity of the antibodies. Urea-glycerol-PAGE has been used to separate the phosphorylated forms of MRLC based on their native charges instead of molecular weight 
(Perrie and Perry, 1970). When a urea-glycerol-PAGE blot of lysate from wild type imaginal discs is probed with the polyclonal antibody against Sqh, three bands are clearly visible (Fig. 1B). From top to bottom, these bands represent nonphosphorylated, monophosphorylated and diphosphorylated forms of Sqh, respectively. In contrast, when these blots are probed with anti-Sqh1P or anti-Sqh2P antibodies, only a single band is revealed (Fig. 1B). To confirm that the single band represents either Sqh1P or Sqh2P specifically, we re-probed these blots with the anti-Sqh antibody and visualized the presence of the other two bands at the expected positions (data not shown). These blots also revealed that less than $20 \%$ of total Sqh proteins are phosphorylated in late larval imaginal discs.

To further demonstrate the specificity of the antibodies against the phosphorylated forms of Sqh, we treated protein samples from wild type imaginal discs with protein phosphatase 1 (PP1) and analyzed the samples by western blotting. PP1 is a general phosphatase that dephosphorylates phospho-Ser and phospho-Thr residues (Cohen, 1989), and thus is predicted to be able to dephosphorylate phospho-Thr20 and phospho-Ser21 on Sqh. On western blots, we observed that the signal of both Sqh1P and Sqh2P decreased significantly after PP1 treatment, whereas the expression of Coracle, a cytoplasmic protein used as a control for protein stability was unaffected during the time course (Fig. 1C, and data not shown).

To demonstrate the specificity of these phospho-specific antibodies to recognize Sqh in Drosophila tissues, we generated mitotic clones of the null sqh allele $s q h^{A X 3}$ in the ovary and immunostained them with antibodies against Sqh1P and Sqh2P. $s q h^{A X 3}$ homozygous mutant clones in the ovarian follicular epithelium showed clearly decreased levels of both Sqh1P and Sqh2P in the mutant cells compared to the neighboring heterozygous cells (Fig. 2). Taken together, these immunoblot and immunostaining experiments clearly demonstrate that the antibodies directed against Sqh1P and Sqh2P are highly specific for the phosphorylated forms of Sqh in Drosophila tissues.

\subsection{Sqh1P and Sqh2P show distinct tissue distributions in wild type embryos}

We next used these antibodies to investigate myosin activation on staged wild type Drosophila embryos, and discovered that the different phosphorylated forms of Sqh show distinct tissue distributions and subcellular localizations. Sqh1P is fairly ubiquitously expressed and has a pattern that is virtually indistinguishable from total Sqh and Zip (Fig. 3A-C; Young et al., 1991; Young et al., 1993). Specifically, Sqh1P can first be detected during cellularization, where it localizes to the leading edge of the cleavage furrows (Fig. 3A). During gastrulation, Sqh1P and Sqh are predominantly found in the cytoplasm, but there is a transient enrichment near the basal plasma membrane (arrows in Fig. 3B) that first disappears in the invaginating cells of the ventral furrow. This pattern of expression is similar to that reported for Zip (myosin heavy chain; (Dawes-Hoang et al., 2005). In the ventral furrow cells, Zip relocalizes to the apical plasma membrane (Dawes-Hoang et al., 2005). Consistent with Sqh (and Sqh1P) working in a complex with Zip in these cells, we occasionally detected an enrichment of Sqh and Sqh1P near the apical plasma membrane of ventral furrow cells (arrowhead in Fig. 3B). It is possible that we could not detect more frequent or robust apical enrichment of Sqh and Sqh1P in ventral furrow cells due to interference from the cytoplasmic expression that persisted in embryos fixed by paraformaldehyde (please see notes on fixation in Experimental Procedures). In subsequent stages, Sqh1P is expressed in nearly all embryonic tissues including the epidermis, foregut, hindgut, midgut, the central nervous system, and the extraembryonic amnioserosa (Fig. 3CG, Fig. 5A-C and data not shown). It is noteworthy that there are some tissues that show substantially higher levels of Sqh1P expression than the surrounding tissues, and that this difference is not reflected in the total Sqh staining. This is most evident in the brain and ventral nerve cord (Fig. 3D and E). In addition, there is an unusual distribution of Sqh1P in 
the dorsal most row of epidermal (DME) cells during dorsal closure (described in more detail below), but this expression is reflected in the total Sqh pattern as well.

Subcellularly, Sqh1P is cytoplasmic but appears to be enriched at cell membranes, thereby producing a cell-outlining pattern (for example Fig. 3C). Sqh1P is enriched apically in the epidermis, salivary gland, foregut and hindgut (Fig. 3C, F, and G), but it is also present along basolateral membranes in these tissues and is noticeably enriched on the basal surface of the hindgut (arrow in Fig. 3G).

Sqh2P, in contrast, is expressed in a limited pattern during embryogenesis. Sqh2P is not detected at all during cellularization or gastrulation (data not shown), and is first noticeably expressed at stage 6 in the cephalic furrow (data not shown), and then later in the invaginating proctodeal and stomodeal openings at stage 9 and 10 (Fig. 4A). It is also strongly expressed in tracheal placodes as they begin to invaginate in stage 11 and continuing throughout tracheal development (Fig. 4B-D and data not shown). We also observe strong Sqh2P expression on the apical surface of the invaginating salivary glands starting at stage 11, although we did not detect strong expression in the salivary gland placode prior to invagination (data not shown). During dorsal closure we noted strong Sqh2P expression in the DME cells, but in a pattern distinct from that of Sqh1P (described in more detail below). Sqh2P is also strongly expressed in the apically constricted cells found at segmental boundaries (Fig. 4D). During head involution, Sqh2P is strongly expressed in the pharynx and foregut (data not shown), as well as in a structure known as the dorsal pouch, which becomes apparent in stage 14 embryos (Fig. 4E). Finally, Sqh2P is expressed in puncta in the epidermis starting at stage 15 in a pattern that presages the epidermal hairs, as well as in other actin rich structures including the posterior spiracles (data not shown). It should be noted that starting in stage 14 embryos, the most obviously staining structures appear to be in the lumen of the tracheal system and the fore- and hindgut. These patterns are non-specific, however, as evidenced by costaining with apical plasma membrane markers such as Crumbs (Crb; Tepass et al., 1990) and Uninflatable (Uif; Zhang and Ward, 2009) that show that the anti-Sqh2P serum predominantly recognizes a luminal component instead of the epidermis (Supplemental Fig. 1).

Subcellularly, Sqh2P is found on the apical membrane. This can be demonstrated most clearly by counterstaining these embryos with antibodies against apically localized proteins. Sqh2P colocalizes with Uif and Crb on the apical surface of the tracheal system and in the dorsal pouch (Figs. 4B-E and data not shown). These co-staining experiments also demonstrate that Sqh2P is upregulated in the segmental boundary cells relative to the rest of the epidermis. Specifically, Uif is expressed at relatively uniform levels at the apical surface of all the epidermal cells including cells of the segmental boundaries where the apical surfaces of adjacent cells are in close proximity (and thus Uif appears to be more strongly expressed here), and at the surface of the intersegmental cells that can be visualized on the ventral surface of the embryo (bracket in Fig. 4D"). Sqh2P, in contrast, is barely detectable in these intersegmental cells while maintaining strong expression in the cells at the segmental boundary (Fig. 4D').

The expression differences between Sqh1P and Sqh2P are most evident in the DME cells during dorsal closure. Midway through embryogenesis, after the germband has retracted, the epidermis covers the ventral and lateral regions of the embryo, leaving a large dorsal hole covered only by a layer of squamous extraembryonic cells known as the amnioserosa. During dorsal closure, the lateral epidermal sheets on both sides of the embryo begin an orchestrated migration toward the midline, where they eventually meet and fuse and thereby close the dorsal surface of the embryo (reviewed in Harden, 2002). During this processes, the DME cells have a special role in organizing these morphogenetic events. Early in dorsal 
closure the DME cells undergo planer polarization in the dorsal-ventral axis resulting in a reorganization of the actin cytoskeleton (Kaltschmidt et al., 2002). These cells subsequently accumulate actin and myosin at the leading edge of these cells, eventually producing an actinomyosin cable through all the DME cells that helps coordinate the migration of the epidermal sheets towards the dorsal midline (Franke et al., 2005; Young et al., 1993). Sqh1P and Sqh2P are both upregulated in the DME cells during dorsal closure, but show a mostly non-overlapping pattern. Starting at stage 13, Sqh1P is strongly expressed in the leading edge of the DME cells in a "bars-on-a-string" distribution (Fig. 5A). This pattern persists during dorsal closure and grows more intense as dorsal closure proceeds (Fig. 5B and C). Similar patterns of expression have been described for both Sqh and Zip (Franke et al., 2005; Wang and Ward, 2010; Young et al., 1993). In contrast to the specific localization of Sqh1P along the actin cable, Sqh2P is strongly upregulated throughout the DME cells during the initiation phase of dorsal closure (stage 13; Fig. 5D), and localizes along the membrane. This expression appears to start in the cells near the anterior end of the row of DME cells and often remains strongest there. We also frequently observe large puncta of Sqh2P expression in these cells (Figs. 5D and E). Beginning in stage 13 embryos, Sqh2P can be found decorating large filopodia that emanate from the dorsal side of the DME cells (Fig. 5D). These filopodia become more numerous during the lateral spreading phase of dorsal closure (stage 14; Fig. 5E), and during the fusion or suturing phase (stage 15; Fig. 5F). It should be noted that Sqh2P also decorates the leading edge of the DME cells, but does so in a more continuous manner than Sqh1P, and does not appear to be enriched in the "bars on a string" pattern found by staining with Sqh1P.

\subsection{Sqh1P and Sqh2P have distinct subcellular localization in imaginal discs}

Sqh1P and Sqh2P are also expressed at high levels in the larval imaginal discs where they also display differential subcellular localizations, as well as slight differences in their expression pattern across the disc epithelium. Confocal microscopy shows that whereas Sqh1P appears to be expressed in all imaginal disc cells (Fig. 6A'), Sqh2P appears to strongly localize to regions of epithelial folds (Fig. 6A"). The epithelial cells of imaginal discs are highly polarized and are interconnected by a junctional complex on the lateral membrane of these cells. From apical to basal, the junction consists of a marginal zone (MZ), adherens junction (AJ) and septate junction (SJ) (Fig. 6B) (reviewed in Badouel and McNeill, 2009; Tepass et al., 2001). The center of a fold in the epithelium represents the juxtaposition of two apical surfaces, with lateral junctions spreading basally on both sides of the fold (schematic drawing in Fig. 6C). Thus, the high expression of Sqh2P in the folds likely reflects a strong apical localization in this tissue. Sqh1P then, appears to have a more basal localization in these cells, but does appear to localize to the apical region of the lateral membrane. In addition, due to the folding of the wing disc, the basal surfaces of imaginal disc cells can be observed in other folds (asterisk in Fig. 6A). In this fold, Sqh1P is expressed at high levels on the basal surface, whereas Sqh2P is barely detectable (Fig. 6A). Note the similarity of the apical and basal expression of Sqh1P in this tissue to that observed in the embryonic epithelia (Fig. 3E and F).

To confirm the differences in subcellular localizations between Sqh1P and Sqh2P on the apical surface of these cells, we examined a confocal Z-series through the depth of an imaginal disc. An XZ projection representing a sagittal cross section through the disc (schematic drawing shown in Fig. 6E) confirms that Sqh2P is more apically localized than Sqh1P. In addition, this sagittal cross section reveals that Sqh2P is expressed in all cells in the imaginal epithelium, although it is more highly expressed in the apically constricted cells lining the fold (arrows in Fig. 6D"). Note the similarity in the expression of Sqh2P in the imaginal disc folds to that of Sqh2P in the cells of the segmental boundary during embryogenesis (Fig. 4D). 
To more firmly define the subcellular localization of Sqh1P and Sqh2P in imaginal discs, we co-stained wing imaginal discs with Sqh1P and Sqh2P and different cellular markers of the lateral membrane. For these experiments we used the septate junction marker Coracle (Cor; Fehon et al., 1994), the adherens junction marker Armadillo (Arm; Peifer and Wieschaus, 1990), and marginal zone maker Crb (Tepass et al., 1990). We found that Sqh1P localizes more apical than Cor, mainly co-localizes with Arm and partially co-localizes with Crb (Fig. 7A-C). In contrast, Sqh2P localizes more apically than both Cor and Arm (Fig. 8B-C). The distribution of Sqh2P appears to primarily co-localize with $\mathrm{Crb}$, although $\mathrm{Crb}$ has a punctate expression in the folds consistent with a marginal zone localization, whereas Sqh2P shows a continuous distribution at the apical ends of these cells, indicating that it is even more apically localized than Crb (Fig. 8A). Thus, Sqh1P mainly localizes to adherens junction with some expression in the marginal zone (Fig. 7D), whereas Sqh2P mainly localizes in the marginal zone and the apical plasma membrane (Fig. 8D).

One of the most interesting observations made with these antibodies is that the different phosphorylated forms of Sqh show distinct subcellular localizations in Drosophila embryos and imaginal discs. Studies in cultured cells have described similar differential subcellular localizations of the monophosphorylated and diphosphorylated forms of MRLC. In resting rat aortic smooth muscle cells, diphosphorylated MRLC was found only on the peripheral regions of the cells, whereas monophosphorylated MRLC was more strongly expressed along fibrillary structures that ran along the long axis of these cells (Sakurada et al., 1998). In motile HeLa cells, the monophosphorylated form of MRLC was found in the cell periphery as well as at the leading edge of the motile cells, whereas the diphosphorylated form was concentrated at the base of the leading edge in a region that was sprouting filopodia, likely associated with focal adhesion complexes (Uchimura et al., 2002). Here we show for the first time that the monophosphorylated and diphosphorylated forms of Sqh also have distinct subcellular localizations in Drosophila cells in an intact organism. In all the Drosophila tissues we observed, both total Sqh and Sqh1P predominantly localizes to the adherens junction, consistent with a role for Sqh in regulating myosin on the cortical actin cytoskeleton. These observations suggest that there is a pool of Sqh in these junctions that is in an equilibrium between nonphosphorylated and monophosphorylated. Likely, cycles of phosphorylation and dephosphorylation occur in situ at the adherens junction to maintain this equilibrium. Sqh1P also localizes to the basal surface of epithelial cells, again consistent with a localization with filamentous actin, and suggesting a pool of Sqh that might be involved in focal adhesion. In contrast, Sqh2P specifically localizes to the very apical surface and in filopodia of imaginal disc cells and a subset of ectodermally-derived epithelial cells in the embryo.

What could account for the different subcellular distribution of Sqh1P and Sqh2P in these tissues? There are at least two, non exclusive possibilities. First, this distribution could be controlled primarily by localized phosphatases. In vitro, phosphorylation of Ser19 on MRLC (Ser21 in Sqh) by myosin light chain kinase (MLCK) occurs 500-fold more readily than phosphorylation on Thr18 (Thr20 in Sqh) (Ikebe and Hartshorne, 1985; Ikebe et al., 1986), suggesting that the equilibrium point in the cell is likely between nonphosphorylated and monophosphorylated, consistent with our observations on western blots from total protein extracts from imaginal discs (Fig. 1). Sakurada et al. (1998) reported that treatment with the protein phosphatase inhibitor, calyculin A, strongly induced diphosphorylation of MRLC in cultured SM-3 smooth muscle cells, arguing that the equilibrium in these cells was being largely regulated by phosphatase activity. Thus an adherens junction localized phosphatase could easily shift the equilibrium point away from diphosphorylated Sqh in the adherens junction, leaving Sqh2P to be found only in places that lack concentration of this phosphatase. In support of this idea is work showing that the myosin binding subunit of both vertebrate and Drosophila myosin phosphatase (MYPT) localizes to the adherens junction 
(Inagaki et al., 1997; Mitonaka et al., 2007). An alternative hypothesis, however, argues that there are unique kinases that show specific subcellular localizations and have a high activity on Sqh that can thereby phosphorylate Sqh mainly to Sqh2P in these locations (Ikebe and Hartshorne, 1985; Ikebe et al., 1986; Ueda et al., 2002). In support of this idea, Sakurada et al. (1998) observed that diphosphorylation of MRLC in rabbit aortic smooth muscle cells is more sensitive to certain protein kinase inhibitors than others, suggesting that different kinases can have differential activities on the phosphorylation state of MRLC (and by extension, Sqh). In addition, MLCK has been shown to have actin binding activity in vertebrate cell, which may account for the higher concentration of diphosphorylated MRLC in actin rich surface features such as lamellipodia and filopodia (Uchimura et al., 2002). There are more than a dozen kinases that have been reported to phosphorylate MRLC (Vicente-Manzanares et al., 2009), but at this point there are no studies showing specific localization of kinases able to phosphorylate Sqh at the apical plasma membrane in Drosophila. Additional studies will be required to address these ideas. For example, if this equilibrium is predominantly maintained by the activity of myosin phosphatase in the adherens junction, then imaginal disc clones of cells mutant for myosin phosphatase should have an upregulation of Sqh2P at the adherens junction.

A potentially more interesting question is what are the functional differences of myosin complexes containing predominately Sqh1P or Sqh2P. As part of the nonmuscle myosin II complex, Sqh serves as the regulatory light chain, affecting the actin-dependent ATPase activity of the complex. Through this activity, myosin can exert motile force along actin filaments. It is well described that phosphorylation of Ser19 on MRLC (Ser21 in Sqh) can greatly increase the actin-dependent ATPase activity of myosin (Ikebe et al., 1988), and that subsequent phosphorylation of Thr18 (Thr20 in Sqh) can further increase this activity (Ikebe and Hartshorne, 1985; Ikebe et al., 1986; Ikebe et al., 1988). The localization of Sqh1P primarily to the adherens junction, a region where filamentous actin bands encircle the cells, suggests that Sqh1P exerts a role primarily in force generation. This activity would be necessary for simple cell shape changes, including apical cell constrictions. The localization of Sqh1P at the leading edge of DME cells during dorsal closure is an excellent example of how this function might work. Previous studies have shown that F-actin forms a continuous cable at the leading edge of the DME cells (Franke et al., 2005; Jacinto et al., 2000; Jacinto et al., 2002a; Jacinto et al., 2002b). The myosin heavy chain (Zip) localizes in a "bars-on-astring" distribution along this actin cable (Franke et al., 2005; Young et al., 1993), identical to what we observed with Sqh1P (Fig. 5). The colocalization of Sqh1P, Zip and actin would then be capable of generating coordinated contractile forces that would close the epidermis around the dorsal hole analogous to the closure of a purse string, and would additionally contribute to the maintenance of a uniform migration front as dorsal closure proceeds (Jacinto et al., 2002a; Kiehart et al., 2000).

What then might be the function of Sqh2P in Drosophila cells? The tissue distribution of Sqh2P may shed some light on this question. During embryogenesis, Sqh2P is strongly expressed primarily in cells that are actively moving, including a number of invaginating tissues such as the salivary glands, the tracheal system, the stomodeum and proctodeum, as well as tissues that coordinate the migration of epithelial sheets including the DME cells during dorsal closure and the dorsal pouch during head involution. The dorsal pouch originates from the most dorsal anterior region of the procephalon and is among the first tissues to involute during head involution. In subsequent stages, the remaining head segments are drawn into the embryo under the dorsal pouch while the dorsal epidermis, led by the dorsal ridge, migrate over the dorsal pouch (Nassif et al., 1998). It is likely that all of these cells that express higher levels of Sqh2P are not typical polarized epithelia, but rather have taken on a pseudo-mesenchymal behavior. As such, the plasma membrane, and in particular the actin cytoskeleton, is more dynamic, producing extensive filopodia and 
lamellipodia. Jacinto et al. (2000) reported that these filopodial protrusions are remarkably dynamic during dorsal closure, and may play an important role in scanning the opposing epithelial cells and for dragging the contralateral cell sheets together. Similarly, it has been shown that the apical regions of imaginal disc cells have long filopodial extensions, and that the disc proper cells are in contact with the peripodial epithelium through these actin-rich membrane extensions (Demontis and Dahmann, 2007; Gibson and Schubiger, 2000). Since these structures are actin-rich surface protrusions, Sqh2P may act as part of a myosin motor carrying cargo to and from the tips of these structures, and as such may play important roles in building these structures and for signaling events occurring in them. There is precedence for myosin activity in filopodial structures. Tokuo et al. (2007) showed that myosin X is crucial for actin reorganization leading to formation of filopodia in African green monkey kidney COS7 cells and NIH3T3 fibroblasts. Myosin X also plays an important role in elongation of filopodia (Berg and Cheney, 2002; Tokuo and Ikebe, 2004). There is no homolog of myosin X in Drosophila (Berg and Cheney, 2002), however. The function of myosin X might therefore be carried out by nonmuscle myosin II in conjunction with Sqh2P. Finally, it is also noteworthy that Sqh2P is first upregulated in the DME cells during the initiation phase of dorsal closure, before migration begins. It is possible that the expression of a Sqh2P-containing myosin complex is a necessary early step in this transition to a pseudo-mesenchymal migratory tissue. The early expression of Sqh2P in the tracheal placodes prior to invagination supports this idea. Finally, although Sqh2P containing myosin complexes may be important for proper control of these cellular processes and developmental events, it appears that it is not strictly essential. Jordan and Karess (1997) used a sqh transgene coding for a nonphosphorylatable Ala in place of Thr20 and found that it could rescue the $s q h^{A X 3}$ null allele to viability. Perhaps there was an increase in the amount of Sqh1P that could compensate for the loss of Sqh2P. Further experiments will need to determine if dorsal closure, tracheal morphogenesis and head involution are less well coordinated or fail in a certain percentage of animals that cannot expresses Sqh2P.

With these antibodies we now have the tools that will enable us to explore a host of cell biological and developmental questions. An antibody directed against the monophosphorylated form of MRLC cross-reacts with Sqh (Matsumura et al., 1998), and has been used to examine myosin activation in a number of developmental systems including planer cell polarity in imaginal discs and the formation and movement of the morphogenetic furrow during adult eye development (Corrigall et al., 2007; Escudero et al., 2007; Winter et al., 2001). With these antibodies, we now have better matched antibodies against both phosphorylated forms of Sqh and thus can investigate a wide range of morphogenetic processes that ultimately require cell shape changes mediated through myosin activation. It will be particularly interesting to dissect the Rho signaling pathway during embryogenesis, as zygotic loss of Rhol specifically affect processes involving tissues that normally express high levels of Sqh2P including dorsal closure and head involution (Magie et al., 1999).

\section{Experimental Procedures}

\subsection{Drosophila strains}

$y w c v s q h^{A X 3}$; $P\{s q h-G F P\}$ and $y w, s q h^{A X 3}, F R T 101 / F M 7 C$ fly lines were generous gifts from Roger Karess (CNRS, France; Jordan and Karess, 1997). Other stocks were obtained from Bloomington Drosophila Stock Center (Bloomington, IN). $w^{1118}$ was used as the wild type strain for the experiments conducted in this study. All Drosophila stocks were maintained on media consisting of corn meal, sugar, yeast, and agar in incubators maintained at a constant temperature of $21^{\circ} \mathrm{C}$ or in a room that typically fluctuated between $21^{\circ} \mathrm{C}$ and $22.5^{\circ} \mathrm{C}$. Genetic experiments were conducted in incubators controlled at a constant temperature of $25^{\circ} \mathrm{C}$. 


\subsection{Generation of antibodies}

To generate phosphorylation site-specific antibodies, two peptides were commercially synthesized corresponding to regions of the Sqh protein with phosphorylated derivatives at Ser21 or Thr20/Ser21. The sequences of the peptides used were Sqh1P: A-Q-R-A-T-S*-NV-F-A-M-C and Sqh2P: A-Q-R-A-T*-S*-N-V-F-A-M-C (* indicates phosphorylated amino acid). The cysteine at the $\mathrm{C}$-terminus of each peptide was added for the conjugation of these peptides to Keyhole limpet hemocyanin (KLH). After conjugation to KLH, the peptides were mixed with Freund's complete adjuvant and used to immunize animals. The Sqh1P peptide was synthesized at Pocono Rabbit Farm and Laboratory Inc. (PRF\&L, Canadensis, PA) and used to immunize guinea pigs. The Sqh2P peptide was synthesized at the Immunological Resource Center at the University of Illinois and used to immunize rats. The animals were boosted according to standard protocols until strong immunoreactivity was observed on immunoblots and on tissue stains. The guinea pig anti-Sqh1P serum was subsequently affinity purified at PRF\&L. Antibodies specific for full-length Sqh are described in Wang and Ward (2010). It should be noted that a commercially available antibody to the monophosphorylated version of vertebrate MRLC differs slightly from the peptide we used for Sqh1P (vertebrate sequence: R-P-Q-R-A-T-S*-N-V-F-A-C)

(Matsumura et al., 1998). We have compared this antibody to the one we generated and found essentially no differences (data not shown).

\subsection{Gel electrophoreses and immunoblotting}

SDS PAGE was performed according to standard procedures (Laemmli, 1970). 25 imaginal discs from wandering $3^{\text {rd }}$ instar larvae were hand dissected in cold fresh PBS. The discs were collected in $1.5 \mathrm{ml}$ centrifuge tubes and homogenized in 1× SDS-PAGE sample buffer ( 1 wing disc per $\mu \mathrm{l}$ buffer) and boiled for $5 \mathrm{~min}$. $20 \mu \mathrm{l}$ samples were loaded in each lane of $12 \%$ polyacrylamide gels and eletrophoresed at $120 \mathrm{~V}$. The proteins on the gel were transferred to PVDF membranes (Bio-Rad Laboratories, Hercules, CA) overnight at $30 \mathrm{~V}$ or for $1 \mathrm{~h}$ at $100 \mathrm{~V}$ at $4{ }^{\circ} \mathrm{C}$ in transfer buffer (25mM Tris, $192 \mathrm{mM}$ Glycine and $20 \%$ methanol) followed by immunoblotting as described below.

Phosphatase treatment of protein samples was done by homogenizing imaginal discs in $1 \times$ PBS with protease inhibitors ( $0.1 \mathrm{mM}$ PMSF, $10 \mu \mathrm{M}$ leupeptin) ( 1.5 wing discs $/ \mu 1 \mathrm{PBS})$, followed by treatment of the disc lysates with $0.025 \mathrm{unit} / \mu \mathrm{l}$ protein phosphatase 1(PP1) (New England Biolabs, Ipswich, MA) for the indicated times.

Urea-glycerol-PAGE and immunoblotting was adapted from Seto et al. (Seto et al., 1990). Imaginal discs from wondering $3^{\text {rd }}$ instar larvae were hand dissected in fresh PBS. $\sim 20$ wing discs plus associated leg and halter discs were homogenized in $20 \mu 1$ urea sample buffer (20mM Tris $\mathrm{pH} 8.6,22 \mathrm{mM}$ glycine, $10 \mathrm{mM}$ DTT, $8 \mathrm{M}$ urea, $0.1 \%$ bromophenol blue). The glycerol-polyacrylamide gels contained $20 \mathrm{mM}$ Tris $\mathrm{pH} 8.6,23 \mathrm{mM}$ glycine, $40 \%$ glycerol, $15 \%$ acrylamide and $0.75 \%$ Bis. The reservoir buffer consisted of $20 \mathrm{mM}$ Tris $\mathrm{pH} 8.6,23 \mathrm{mM}$ glycine, $1 \mathrm{mM}$ sodium thioglycolate, and $1 \mathrm{mM}$ DTT. The gels were pre-electrophoresed for $1 \mathrm{~h}$ at $400 \mathrm{~V}$ in $4^{\circ} \mathrm{C}$. The disc samples were then loaded onto the gel and eletrophoresed for 5 $\mathrm{h}$ at $400 \mathrm{~V}$ in $4^{\circ} \mathrm{C}$. The proteins on the gel were transferred to PVDF membranes (Bio-Rad Laboratories, Hercules, CA) overnight at $30 \mathrm{~V}\left(4^{\circ} \mathrm{C}\right)$ in transfer buffer containing $20 \mathrm{mM}$ Tris (pH 7.5) and $20 \%$ methanol followed by immunoblotting as described below.

Immunoblotting of all blots were blocked in 1XTBS (0.1M NaCl, 0.02M Tris, pH7.5 7.6) plus $0.1 \%$ Tween 20 and 5\% nonfat dry milk (blocking buffer) for $1 \mathrm{~h}$ in room temperature, then incubated with primary antibodies in blocking buffer overnight at $4{ }^{\circ} \mathrm{C}$. After three quick rinses and three 15-min washes with 1XTBS plus $0.1 \%$ Tween 20 , the blots were incubated with HRP-conjugated secondary antibodies in blocking buffer for $2-3 \mathrm{~h}$ at room 
temperature. After three quick rinses and three 15-min washes with 1XTBS plus $0.1 \%$ Tween20, the blots were then developed with SuperSignal West Pico Chemiluminescent Substrate (Thermo Fisher Scientific Inc., Rockford, IL) for $1 \mathrm{~min}$. Chemiluminescent signals were detected on a KODAC imaging station or exposed to X-ray film, developed and scanned.

The following antibodies were used at indicated dilutions: mouse anti-Sqh (1:5000); affinity purified guinea pig anti-Sqh1P (1:1000); rat anti-Sqh1P (1:3000); mouse anti-Coracle (1:1000) (Fehon et al., 1994). HRP-conjugated secondary antibodies (Jackson ImmunoResearch Laboratories Inc., West Grove, PA) were used at 1:5000.

\subsection{Immunohistochemistry}

Imaginal discs were hand dissected from wandering $3^{\text {rd }}$ instar larvae or prepupae in fresh PBS, and fixed immediately in $4 \%$ paraformaldehyde for $20 \mathrm{~min}$. For embryos, we tested three different fixation methods, as well as combinations of the three. We found that $4 \%$ paraformaldehyde fixation for $20 \mathrm{~min}$ at room temperature (Fehon et al., 1994) preserved the tissue morphology and gave the best staining for all three antibodies. $10 \%$ ice-cold trichloroacetic acid fixation for 20 minutes appeared to preserve dynamic membrane structures well, and preserved the epitope for Sqh2P, possibly owing to its ability to inhibit phosphatases (Hayashi et al., 1999), but did not permit efficient staining of Sqh or Sqh1P. Similarly, neither Sqh, Sqh1P nor Sqh2P stained well in embryos fixed using a heatmethanol procedure (attempted for staining gastrulating embryos; Muller and Wieschaus, 1996). Therefore, all of the embryos shown here were fixed using $4 \%$ paraformaldehyde for 20 minutes. The following antibodies were used at the given dilutions: mouse anti-Sqh 1:3000; guinea pig anti-Sqh1P 1:1000 (or affinity purified guinea pig anti-Sqh1P 1:500); rat anti-Sqh2P 1:2000; mouse anti-Crumbs 1:100 (clone Cq4 from Developmental Studies Hybridoma Bank at the University of Iowa); mouse anti-Armadillo 1:100 (clone N27A1, DSHB); mouse anti-Coracle 1:400 (gift from Richard Fehon, University of Chicago; Fehon et al., 1994). Secondary antibodies were obtained from Jackson ImmunoResearch Laboratories (West Grove, PA) and used at 1:400. Confocal images were acquired on a Zeiss LSM510 Meta Laser Scanning Confocal Microscope (Carl Zeiss Inc, Thornwood NY). Photomicrographs were adjusted for brightness and contrast with the Adobe Photoshop (version CS3, San Jose, CA), and Figures were complied in Adobe Illustrator (version CS3, San Jose, CA).

\subsection{Mosaic clones}

The FLP/FRT system (Xu and Rubin, 1993) was used to generate $s q h^{A X 3}$ homozygous mutant clones in the ovary. To generate sqh clones, $y w, U b i-G F P, F R T 101 / Y ; h s F L P /+$ males were crossed to $y w, s q h^{A X 3}, F R T 101 / F M 7 C$ virgin females. Then, $y w$, ubiGFP, FRT101/yw, $s q h^{A X 3}, F R T 101 ; h s P L F /+2-3$ day old females were selected and heat shocked at $37^{\circ} \mathrm{C}$ for $1 \mathrm{~h}$. Ovaries were dissected $24 \mathrm{~h}$ after heat shock, and fixed and stained as described above. Homozygous sqh mutant cells are marked by the absence of ubiquitous GFP.

\section{Supplementary Material}

Refer to Web version on PubMed Central for supplementary material.

\section{Acknowledgments}

We thank Carl Thummel (University of Utah), in whose lab antibodies against Sqh2P were generated. We thank Michelle Wickersheim and Shannon Stewart for technical help with the testing of the Sqh and Sqh1P sera, and Courtney Bone for technical help with fixing and mounting gastrulating embryos. We thank the developmental 
studies hybridoma bank for antibodies used in this study. We thank Roger Karess for the $s q h^{A X 3}$ strains used in these studies. We thank Brooke McCartney and Shi Mulinari for insightful discussions about the study. The project was supported by NIH Grant Number P20 RR15563 and NIH Grant Number R01HD047570 from the National Center for Research Resources.

\section{References}

Badouel C, McNeill H. Apical junctions and growth control in Drosophila. Biochim. Biophys. Acta 2009;1788:755-760. [PubMed: 18952051]

Berg JS, Cheney RE. Myosin-X is an unconventional myosin that undergoes intrafilopodial motility. Nat. Cell Biol 2002;4:246-250. [PubMed: 11854753]

Bosgraaf L, van Haastert PJ. The regulation of myosin II in Dictyostelium. Eur. J. Cell Biol 2006;85:969-979. [PubMed: 16814425]

Cohen P. The structure and regulation of protein phosphatases. Annu. Rev. Biochem 1989;58:453508. [PubMed: 2549856]

Conti MA, Adelstein RS. Nonmuscle myosin II moves in new directions. J. Cell Sci 2008;121:11-18. [PubMed: 18096687]

Corrigall D, Walther RF, Rodriguez L, Fichelson P, Pichaud F. Hedgehog signaling is a principal inducer of Myosin-II-driven cell ingression in Drosophila epithelia. Dev. Cell 2007;13:730-742. [PubMed: 17981140]

Dawes-Hoang RE, Parmar KM, Christiansen AE, Phelps CB, Brand AH, Wieschaus EF. folded gastrulation, cell shape change and the control of myosin localization. Development 2005;132:4165-4178. [PubMed: 16123312]

Demontis F, Dahmann C. Apical and lateral cell protrusions interconnect epithelial cells in live Drosophila wing imaginal discs. Dev. Dyn 2007;236:3408-3418. [PubMed: 17854054]

Edwards KA, Kiehart DP. Drosophila nonmuscle myosin II has multiple essential roles in imaginal disc and egg chamber morphogenesis. Development 1996;122:1499-1511. [PubMed: 8625837]

Escudero LM, Bischoff M, Freeman M. Myosin II regulates complex cellular arrangement and epithelial architecture in Drosophila. Dev. Cell 2007;13:717-729. [PubMed: 17981139]

Fehon RG, Dawson IA, Artavanis-Tsakonas S. A Drosophila homologue of membrane-skeleton protein 4.1 is associated with septate junctions and is encoded by the coracle gene. Development 1994;120:545-557. [PubMed: 8162854]

Franke JD, Montague RA, Kiehart DP. Nonmuscle myosin II generates forces that transmit tension and drive contraction in multiple tissues during dorsal closure. Curr. Biol 2005;15:2208-2221. [PubMed: 16360683]

Gibson MC, Schubiger G. Peripodial cells regulate proliferation and patterning of Drosophila imaginal discs. Cell 2000;103:343-350. [PubMed: 11057906]

Gotwals PJ, Fristrom JW. Three neighboring genes interact with the Broad-Complex and the Stubblestubbloid locus to affect imaginal disc morphogenesis in Drosophila. Genetics 1991;127:747-759. [PubMed: 1903118]

Halsell SR, Kiehart DP. Second-site noncomplementation identifies genomic regions required for Drosophila nonmuscle myosin function during morphogenesis. Genetics 1998;148:1845-1863. [PubMed: 9560399]

Harden N. Signaling pathways directing the movement and fusion of epithelial sheets: lessons from dorsal closure in Drosophila. Differentiation 2002;70:181-203. [PubMed: 12147138]

Hartshorne DJ. Myosin phosphatase: subunits and interactions. Acta. Physiol. Scand 1998;164:483493. [PubMed: 9887971]

Hayashi K, Yonemura S, Matsui T, Tsukita S. Immunofluorescence detection of ezrin/radixin/moesin (ERM) proteins with their carboxyl-terminal threonine phosphorylated in cultured cells and tissues. J. Cell Sci 1999;112(Pt 8):1149-1158. [PubMed: 10085250]

Ikebe M, Hartshorne DJ. Phosphorylation of smooth muscle myosin at two distinct sites by myosin light chain kinase. J. Biol. Chem 1985;260:10027-10031. [PubMed: 3839510] 
Ikebe M, Hartshorne DJ, Elzinga M. Identification, phosphorylation, and dephosphorylation of a second site for myosin light chain kinase on the 20,000-dalton light chain of smooth muscle myosin. J. Biol. Chem 1986;261:36-39. [PubMed: 3079756]

Ikebe M, Koretz J, Hartshorne DJ. Effects of phosphorylation of light chain residues threonine 18 and serine 19 on the properties and conformation of smooth muscle myosin. J. Biol. Chem 1988;263:6432-6437. [PubMed: 2966156]

Inagaki N, Nishizawa M, Ito M, Fujioka M, Nakano T, Tsujino S, Matsuzawa K, Kimura K, Kaibuchi $\mathrm{K}$, Inagaki M. Myosin binding subunit of smooth muscle myosin phosphatase at the cell-cell adhesion sites in MDCK cells. Biochem. Biophys. Res. Commun 1997;230:552-556. [PubMed: 9015360]

Jacinto A, Wood W, Balayo T, Turmaine M, Martinez-Arias A, Martin P. Dynamic actin-based epithelial adhesion and cell matching during Drosophila dorsal closure. Curr. Biol 2000;10:14201426. [PubMed: 11102803]

Jacinto A, Wood W, Woolner S, Hiley C, Turner L, Wilson C, Martinez-Arias A, Martin P. Dynamic analysis of actin cable function during Drosophila dorsal closure. Curr. Biol 2002a;12:1245-1250. [PubMed: 12176336]

Jacinto A, Woolner S, Martin P. Dynamic analysis of dorsal closure in Drosophila: from genetics to cell biology. Dev. Cell 2002b;3:9-19. [PubMed: 12110163]

Jordan P, Karess R. Myosin light chain-activating phosphorylation sites are required for oogenesis in Drosophila. J. Cell Biol 1997;139:1805-1819. [PubMed: 9412474]

Kaltschmidt JA, Lawrence N, Morel V, Balayo T, Fernandez BG, Pelissier A, Jacinto A, Martinez Arias A. Planar polarity and actin dynamics in the epidermis of Drosophila. Nat. Cell Biol 2002;4:937-944. [PubMed: 12447392]

Karess RE, Chang XJ, Edwards KA, Kulkarni S, Aguilera I, Kiehart DP. The regulatory light chain of nonmuscle myosin is encoded by spaghetti-squash, a gene required for cytokinesis in Drosophila. Cell 1991;65:1177-1189. [PubMed: 1905980]

Kiehart DP, Galbraith CG, Edwards KA, Rickoll WL, Montague RA. Multiple forces contribute to cell sheet morphogenesis for dorsal closure in Drosophila. J. Cell Biol 2000;149:471-490. [PubMed: 10769037]

Kosako H, Yoshida T, Matsumura F, Ishizaki T, Narumiya S, Inagaki M. Rho-kinase/ROCK is involved in cytokinesis through the phosphorylation of myosin light chain and not ezrin/radixin/ moesin proteins at the cleavage furrow. Oncogene 2000;19:6059-6064. [PubMed: 11146558]

Laemmli UK. Cleavage of structural proteins during the assembly of the head of bacteriophage T4. Nature 1970;227:680-685. [PubMed: 5432063]

Lee JH, Koh H, Kim M, Kim Y, Lee SY, Karess RE, Lee SH, Shong M, Kim JM, Kim J, Chung J. Energy-dependent regulation of cell structure by AMP-activated protein kinase. Nature 2007;447:1017-1020. [PubMed: 17486097]

Magie CR, Meyer MR, Gorsuch MS, Parkhurst SM. Mutations in the Rho1 small GTPase disrupt morphogenesis and segmentation during early Drosophila development. Development 1999;126:5353-5364. [PubMed: 10556060]

Matsumura F, Ono S, Yamakita Y, Totsukawa G, Yamashiro S. Specific localization of serine 19 phosphorylated myosin II during cell locomotion and mitosis of cultured cells. J. Cell Biol 1998;140:119-129. [PubMed: 9425160]

Mitonaka T, Muramatsu Y, Sugiyama S, Mizuno T, Nishida Y. Essential roles of myosin phosphatase in the maintenance of epithelial cell integrity of Drosophila imaginal disc cells. Dev. Biol 2007;309:78-86. [PubMed: 17662709]

Muller HA, Wieschaus E. armadillo, bazooka, and stardust are critical for early stages in formation of the zonula adherens and maintenance of the polarized blastoderm epithelium in Drosophila. J. Cell Biol 1996;134:149-163. [PubMed: 8698811]

Nassif C, Daniel A, Lengyel JA, Hartenstein V. The role of morphogenetic cell death during Drosophila embryonic head development. Dev. Biol 1998;197:170-186. [PubMed: 9630744]

Peifer M, Wieschaus E. The segment polarity gene armadillo encodes a functionally modular protein that is the Drosophila homolog of human plakoglobin. Cell 1990;63:1167-1176. [PubMed: 2261639] 
Perrie WT, Perry SV. An electrophoretic study of the low-molecular-weight components of myosin. Biochem. J 1970;119:31-38. [PubMed: 5485752]

Sakurada K, Seto M, Sasaki Y. Dynamics of myosin light chain phosphorylation at Ser19 and Thr18/ Ser19 in smooth muscle cells in culture. Am. J. Physiol 1998;274:C1563-C1572. [PubMed: 9611121]

Seto M, Sasaki Y, Sasaki Y. Alteration in the myosin phosphorylation pattern of smooth muscle by phorbol ester. Am. J. Physiol 1990;259:C769-C774. [PubMed: 2240194]

Tan JL, Ravid S, Spudich JA. Control of nonmuscle myosins by phosphorylation. Annu. Rev. Biochem 1992;61:721-759. [PubMed: 1497323]

Tepass U, Tanentzapf G, Ward R, Fehon R. Epithelial cell polarity and cell junctions in Drosophila. Annu. Rev. Genet 2001;35:747-784. [PubMed: 11700298]

Tepass U, Theres C, Knust E. crumbs encodes an EGF-like protein expressed on apical membranes of Drosophila epithelial cells and required for organization of epithelia. Cell 1990;61:787-799. [PubMed: 2344615]

Tokuo H, Ikebe M. Myosin X transports Mena/VASP to the tip of filopodia. Biochem. Biophys. Res. Commun 2004;319:214-220. [PubMed: 15158464]

Tokuo H, Mabuchi K, Ikebe M. The motor activity of myosin-X promotes actin fiber convergence at the cell periphery to initiate filopodia formation. J. Cell Biol 2007;179:229-238. [PubMed: 17954606]

Uchimura T, Fumoto K, Yamamoto Y, Ueda K, Hosoya H. Spatial Localization of Mono-and Diphosphorylated Myosin II Regulatory Light Chain at the Leading Edge of Motile HeLa Cells. Cell Structure and Function 2002;27:479-486. [PubMed: 12576640]

Ueda K, Murata-Hori M, Tatsuka M, Hosoya H. Rho-kinase contributes to diphosphorylation of myosin II regulatory light chain in nonmuscle cells. Oncogene 2002;21:5852-5860. [PubMed: 12185584]

Vereshchagina N, Bennett D, Szoor B, Kirchner J, Gross S, Vissi E, White-Cooper H, Alphey L. The essential role of PP1beta in Drosophila is to regulate nonmuscle myosin. Mol. Biol Cell 2004;15:4395-4405. [PubMed: 15269282]

Vicente-Manzanares M, Ma X, Adelstein RS, Horwitz AR. Non-muscle myosin II takes centre stage in cell adhesion and migration. Nat. Rev. Mol. Cell Biol 2009;10:778-790. [PubMed: 19851336]

Wang X, Ward RE. Sec61alpha is required for dorsal closure during Drosophila embryogenesis through its regulation of Dpp signaling. Dev. Dyn 2010;239:784-797. [PubMed: 20112345]

Winter CG, Wang B, Ballew A, Royou A, Karess R, Axelrod JD, Luo L. Drosophila Rho-associated kinase (Drok) links Frizzled-mediated planar cell polarity signaling to the actin cytoskeleton. Cell 2001;105:81-91. [PubMed: 11301004]

Xu T, Rubin GM. Analysis of genetic mosaics in developing and adult Drosophila tissues. Development 1993;117:1223-1237. [PubMed: 8404527]

Yamashiro S, Totsukawa G, Yamakita Y, Sasaki Y, Madaule P, Ishizaki T, Narumiya S, Matsumura F. Citron kinase, a Rho-dependent kinase, induces diphosphorylation of regulatory light chain of myosin II. Mol. Biol. Cell 2003;14:1745-1756. [PubMed: 12802051]

Young PE, Pesacreta TC, Kiehart DP. Dynamic changes in the distribution of cytoplasmic myosin during Drosophila embryogenesis. Development 1991;111:1-14. [PubMed: 1901784]

Young PE, Richman AM, Ketchum AS, Kiehart DP. Morphogenesis in Drosophila requires nonmuscle myosin heavy chain function. Genes Dev 1993;7:29-41. [PubMed: 8422986]

Zhang L, Ward RE. uninflatable encodes a novel ectodermal apical surface protein required for tracheal inflation in Drosophila. Dev. Biol 2009;336:201-212. [PubMed: 19818339] 

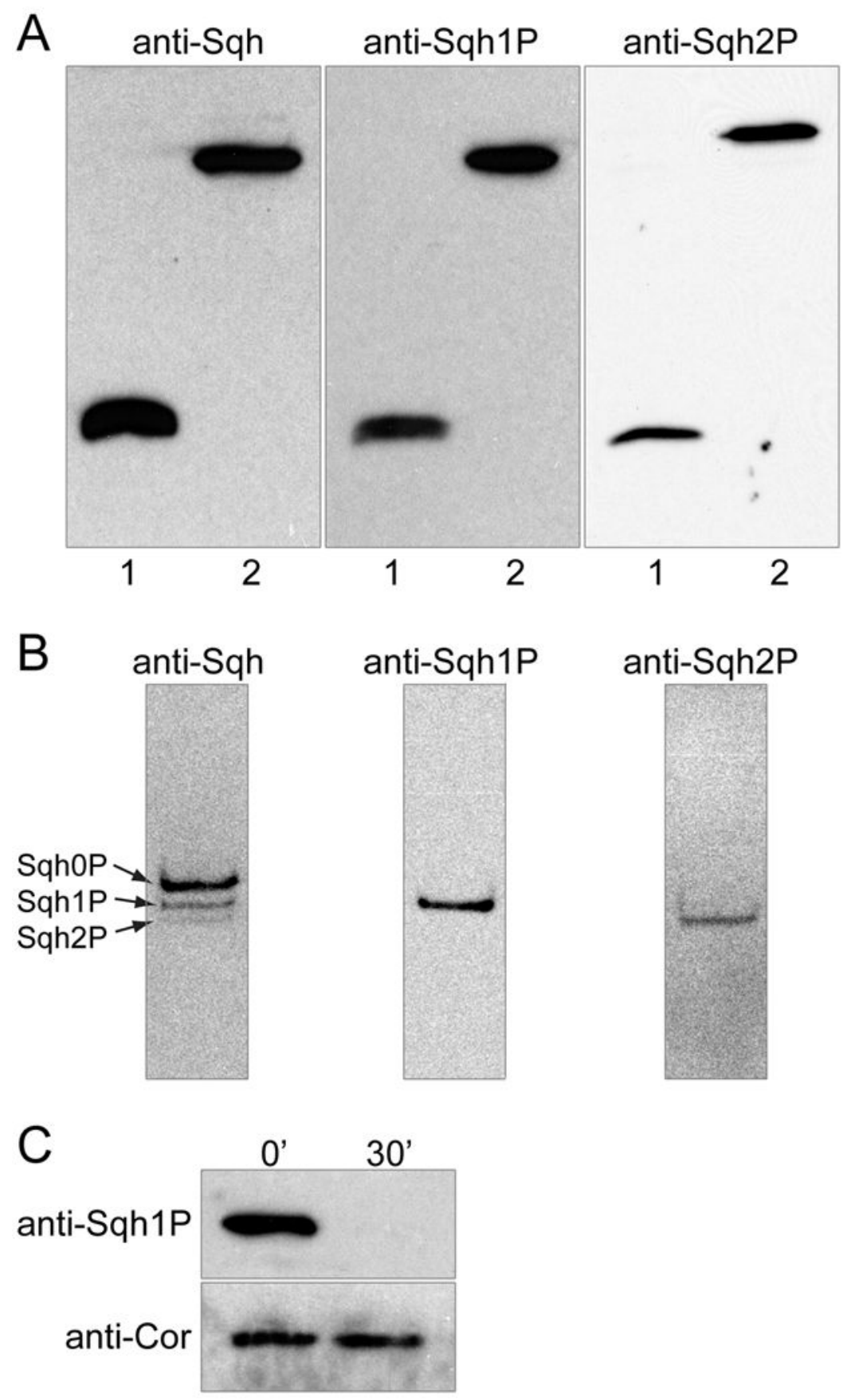

Fig. 1. Antibodies against Sqh1P and Sqh2P are specific

(A) Western immunoblots incubated with anti-Sqh, anti-Sqh1P or anti-Sqh2P antibodies. Lane 1 of each blot contains protein lysate extracted from imaginal discs of $w^{1118}$ (wild type) $3^{\text {rd }}$ instar larvae. Lane 2 of each blot contains lysate extracted from imaginal discs of $y w c v s q h^{A x 3} ; P\{s q h-G F P\} 3^{\text {rd }}$ instar larvae. The bottom band is $\sim 21-\mathrm{kD}$ on each blot, whereas the top band is $\sim 48-\mathrm{kD}$. (B) Western immunoblots of $w^{1118} 3^{\text {rd }}$ instar larval lysates separated by urea-glycerol-PAGE. Anti-Sqh antiserum detects three protein forms (arrows) corresponding to nonphosphorylated, monophosphorylated and diphosphorylated Sqh (from top to bottom). Antibodies against Sqh1P or anti-Sqh2P detect only a single protein species. (C) Phosphatase treatment of $w^{1118} 3^{\text {rd }}$ instar larval lysates reveals that the antibodies 
against Sqh1P specifically recognize phosphorylated protein. Protein samples were treated with PP1 for $30 \mathrm{~min}$. Coracle was used as a control for loading and transfer. 

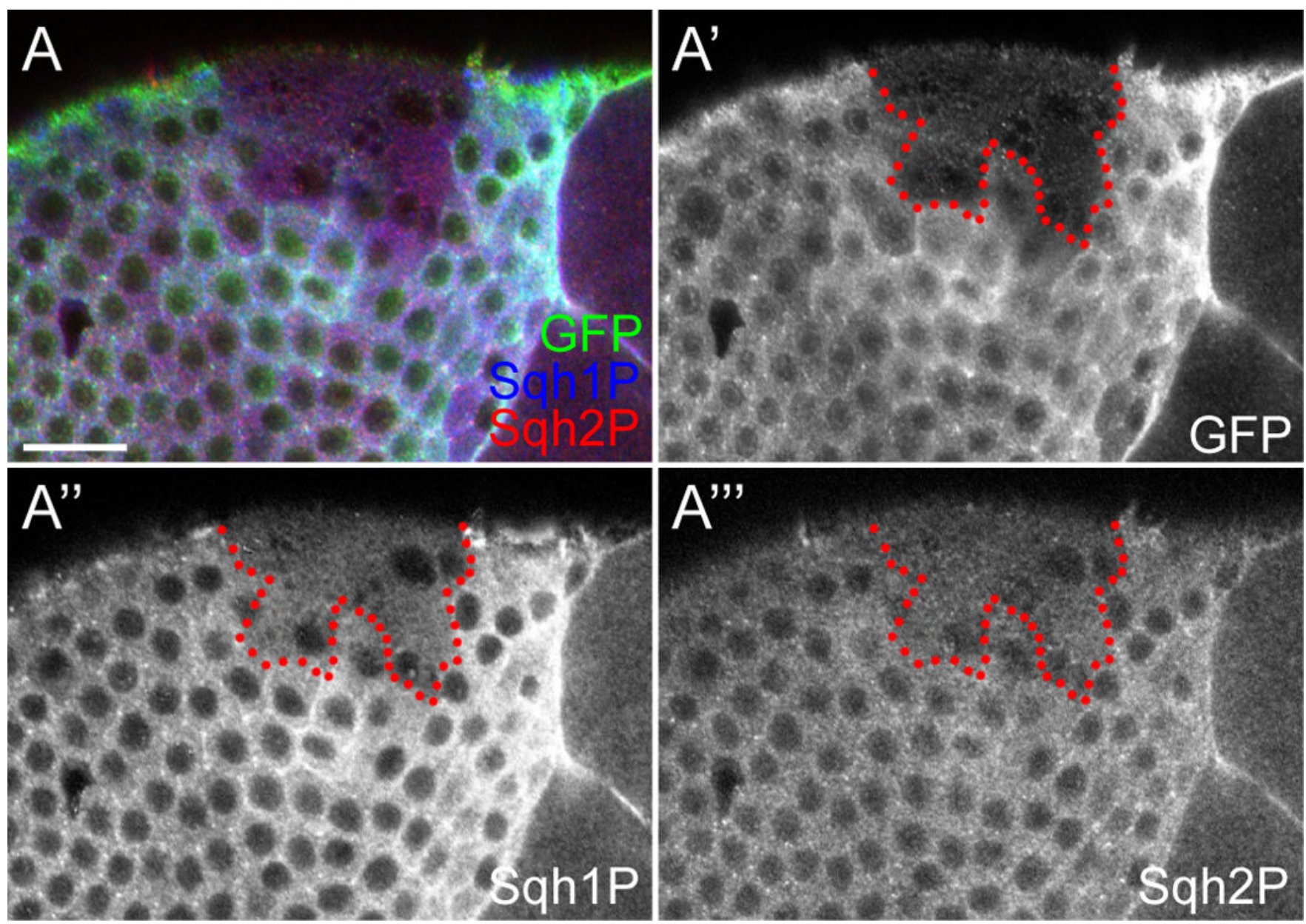

Fig. 2. Sqh1P and Sqh2P levels are strongly reduced in follicle cell clones of $s q h^{A X 3}$

(A) Confocal optical section of the surface of the follicular epithelium in an ovary stained with antibodies against GFP (green, and as single channel in A'), Sqh1P (blue, and as single channel in A") and Sqh2P (red, and as single channel in A"') antibodies. Red dotted lines indicate the boundary between mutant and wild type cells. The absence of GFP indicates a clone of cells homozygous for the null allele $s q h^{A X 3}$. Note that Sqh1P and Sqh2P are strongly reduced in the mutant cells. (Scale bars $=20 \mu \mathrm{m}$ ) 

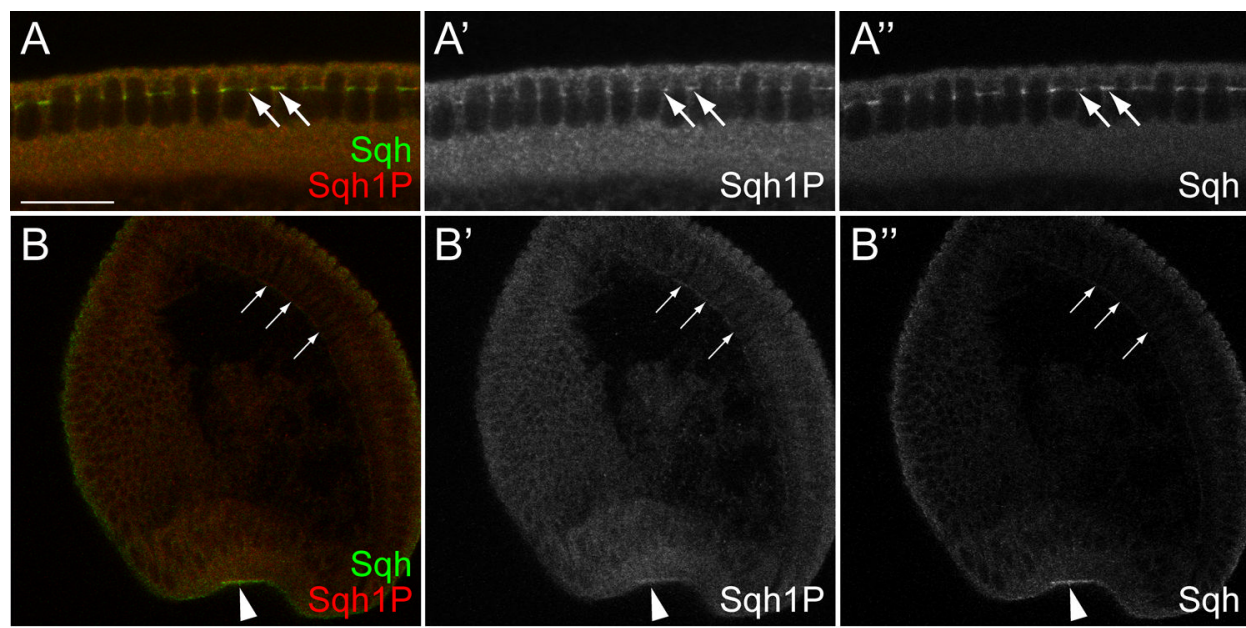

B'
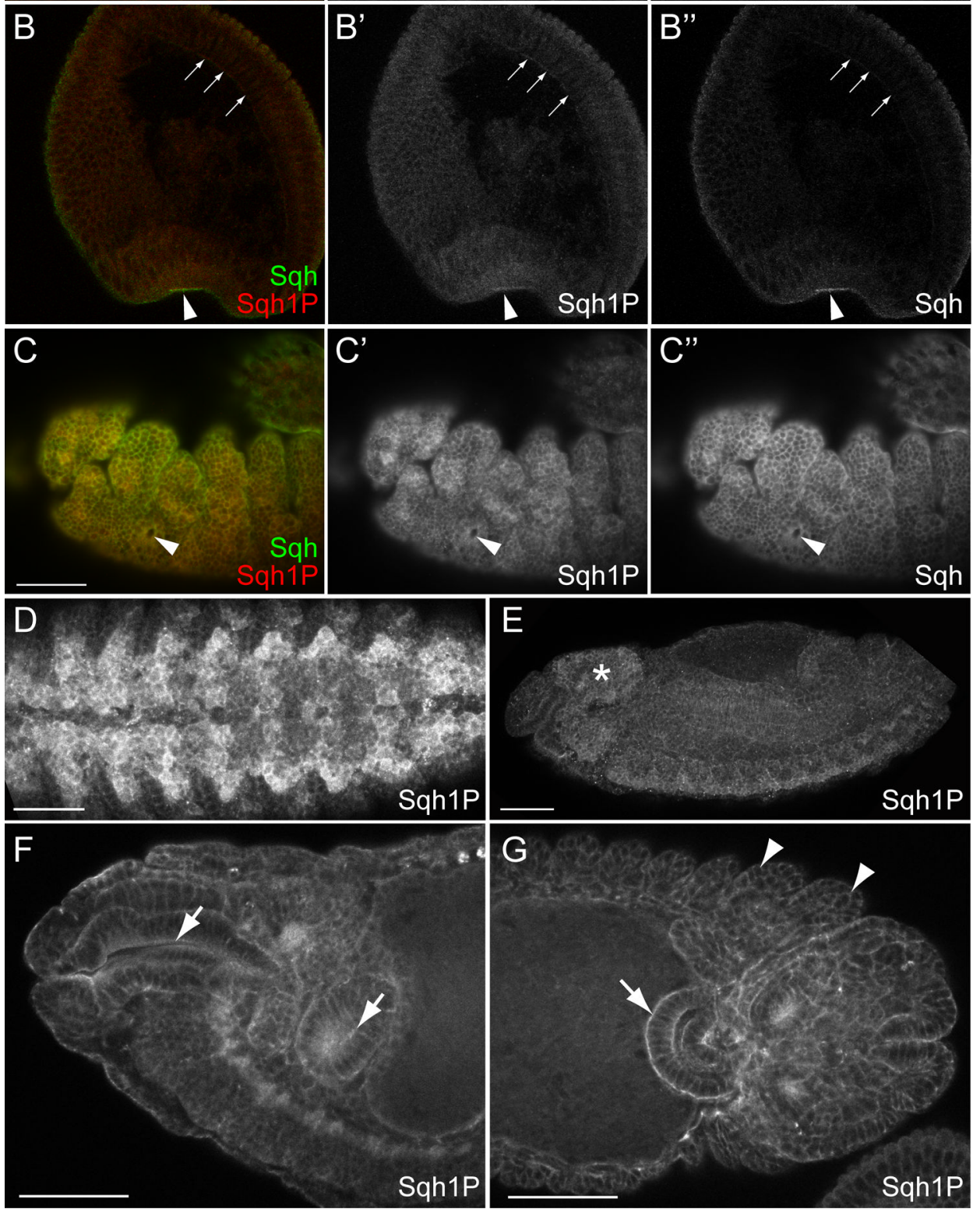

Fig. 3. The expression pattern of Sqh1P in staged embryos

Confocal optical sections of wild type embryos stained with antibodies against Sqh1P and total Sqh. All embryos are displayed with anterior to the left. (A) A confocal optical section through the middle of a cellularizing embryo stained with antibodies against Sqh1P (red and as single channel in A') and total Sqh (green and as single channel in A"). Note the staining of both Sqh and Sqh1P at the leading edge of the cleavage furrows (arrows). (B) Confocal optical section of a wild type gastrulating embryo in cross section stained with antibodies against Sqh1P (red and as single channel in B') and total Sqh (green and as single channel in B"). Sqh and Sqh1P are observed in the cytoplasm with a slight enrichment near the basal membrane in dorsal and lateral cells (arrows), with less basal enrichment and more apical 
enrichment on gastrulating cells (arrowhead). (C) An open pinhole confocal micrograph of the anterior end of a stage 12 embryo stained with antibodies against Sqh1P (red and as single channel in C') and total Sqh (green and as single channel in C"). Note the similarity of the staining, and that both Sqh and Sqh1P appear to outline epithelial cells. The arrowhead indicates the involuting salivary gland and shows that Sqh and Sqh1P are localized apically (towards the lumen of the salivary gland). (D) Ventral view of a stage 14 embryo stained with antibodies against Sqh1P showing high levels of expression in the ventral nerve cord. (E) Confocal optical section of a stage 14 wild type embryo stained with antibodies against Sqh1P reveal that it is noticeably upregulated in the brain (asterisk) and ventral nerve cord relative to the rest of the embryo. (F) Sqh1P is strongly expressed in the pharynx and foregut of a stage 14 embryo, where it is enriched apically (arrows). (G) Sqh1P is also expressed at high levels in the hindgut (arrow) and epidermis (arrowhead) of a stage 14 embryo. Note that the expression in the hindgut is on both the apical and basal surfaces (arrow indicates the basal surface of the hindgut) of these cells. (Scale bars $=20 \mu \mathrm{m}$ in A, $50 \mu \mathrm{m}$ in $\mathrm{C}-\mathrm{G})$ 


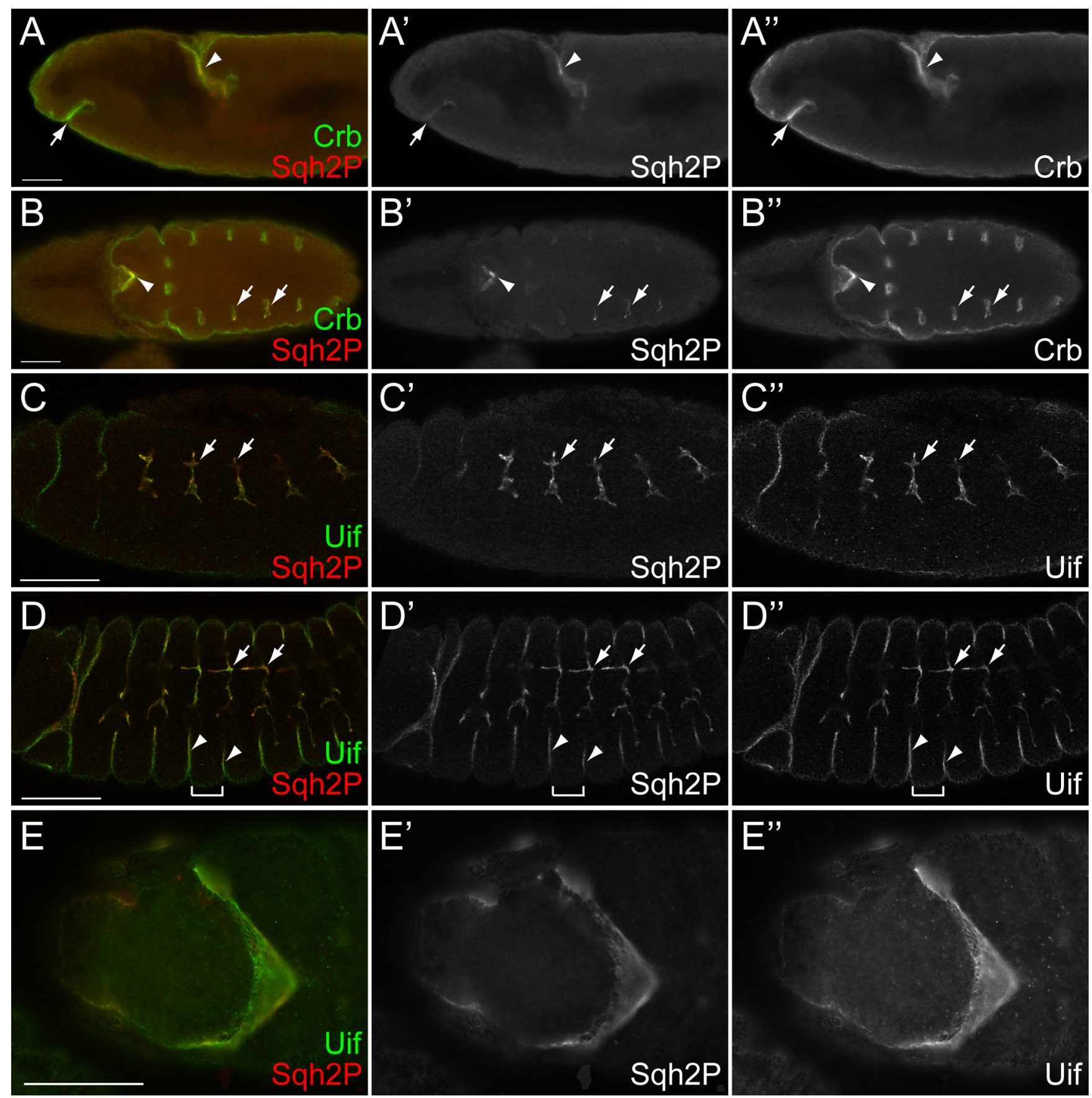

Fig. 4. The expression pattern of Sqh2P in staged embryos

Confocal optical sections of wild type embryos stained with antibodies against Sqh2P (red and as single channel in ') and either Crb (green and as single channel in A" and B") or Uif (green and in single channel in C"-E"). All embryos are displayed with anterior to the left and dorsal up (or facing in B and E). (A) In a stage 10 embryo, Sqh2P is expressed in the invaginating stomodeum (arrow) and proctodeum (arrowhead). (B) In a stage 11 embryo, $\mathrm{Sqh} 2 \mathrm{P}$ is strongly expressed in the tracheal placodes (arrows), and can still be observed in the proctodeal opening (arrowhead). (C) In a stage 12 embryo, Sqh2P is expressed in the invaginating tracheal metameres (arrows). (D) In a stage 13 embryo, Sqh2P is still expressed in the tracheal system as adjacent metameres are fusing (arrows). In addition, Sqh2P is 
strongly expressed in epidermal cells that line the segmental boundary (arrowheads), but is not highly expressed in the intersegmental epidermal cells (denoted by bracket). In contrast, Uif is expressed in both the segmental boundary cells and the intersegmental epidermal cells. (E) Open pinhole confocal micrograph of a stage 14 embryo showing strong localization of Sqh2P in the dorsal pouch. In all the panels note the colocalization of Sqh2P with $\mathrm{Crb}$ and Uif on the apical surface of these cells. (Scale bars $=50 \mu \mathrm{m})$ 

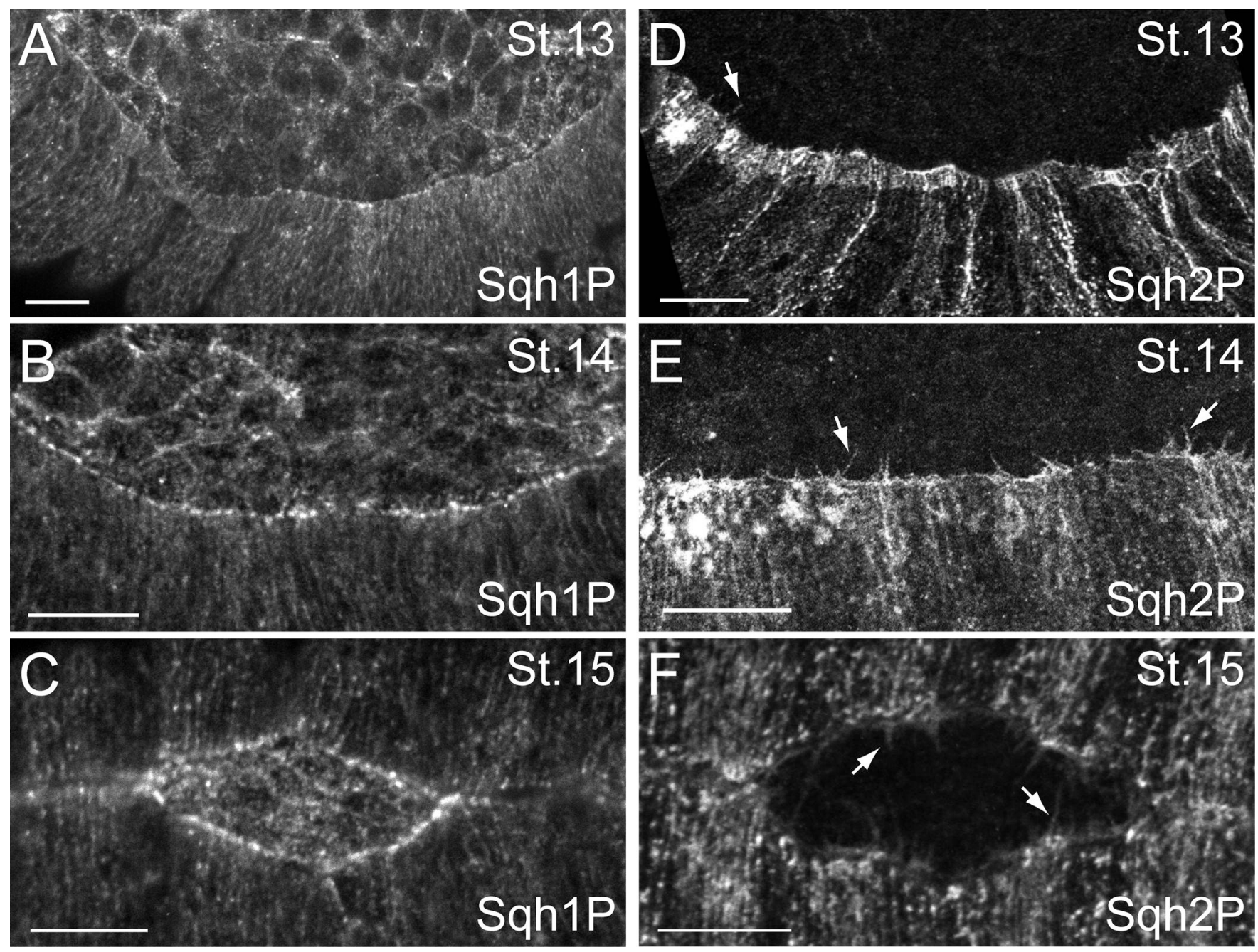

Fig. 5. Sqh1P and Sqh2P have distinct distributions in the DME cells during dorsal closure (A-C) Confocal optical sections of stage 13-15 $w^{1118}$ embryos stained with antibodies against Sqh1P. At all stages, Sqh1P is strongly upregulated at the leading edge of the DME cells, and localizes in a "bars-on-a-string" distribution at this leading edge. This distribution of Sqh1P is identical to the pattern observed with antibodies against Sqh and Zip and is consistent with a 34 localization on the actinomyosin cable that has been well described in these cells during dorsal closure. (D-F) Confocal optical sections of stage 13-15 $w^{1118}$ embryos stained with antibodies against Sqh2P. At stage 13 (D), Sqh2P is strongly upregulated throughout the DME cells. Large aggregates of Sqh2P are often seen in these cells, particularly near the anterior end of the DME cells. Long filopodia that express Sqh2P become apparent at this stage as well (arrow). During stages $14-15$, Sqh2P is strongly expressed at the leading edge of the DME cells and in filopodial protrusions (arrows), although the distribution along the leading edge is continuous and does not correlate with the "bars-on-a-string" distribution seen with Sqh1P. All embryos are displayed with anterior to the left. (Scale bars $=20 \mu \mathrm{m})$ 

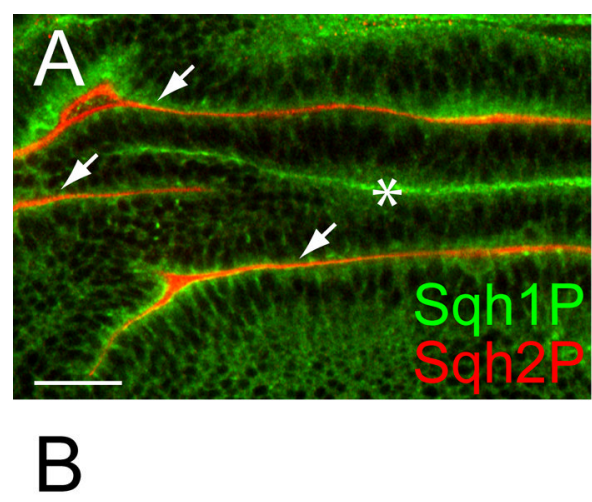

$M Z$ AJ [ SJ
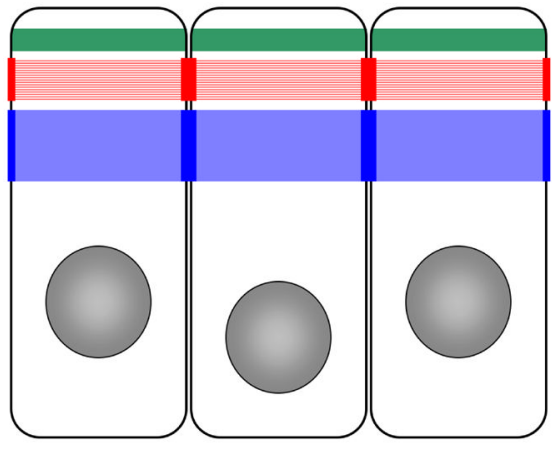

apical
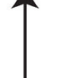

basal
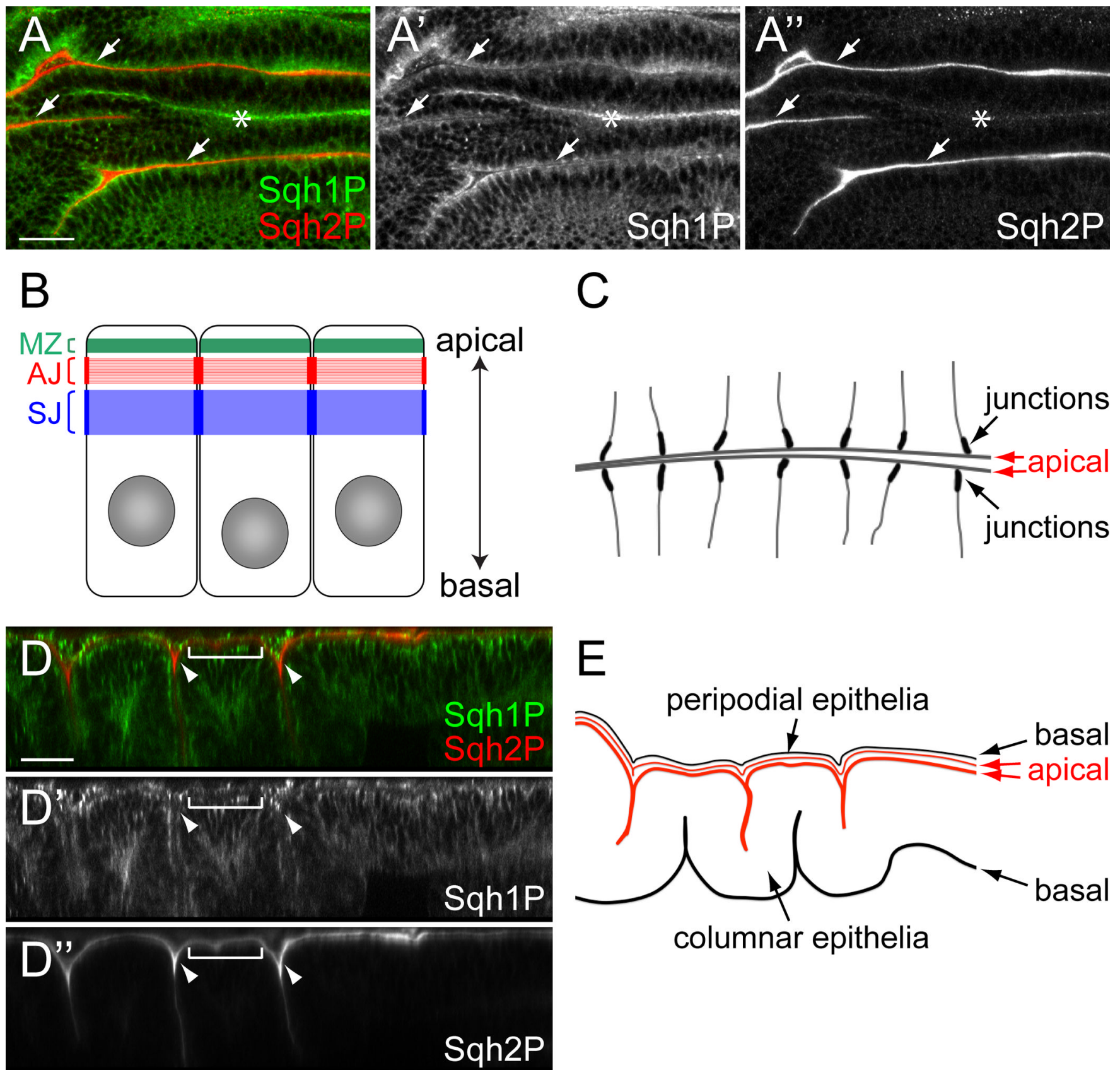
can then be visualized on both sides of the fold. (D-D") XZ projection of a Z-stack confocal image of a wing imaginal disc double labeled with antibodies against Sqh1P (green, and as single channel in D') and Sqh2P (red, and as single channel in D"). Note that Sqh2P is more apical than Sqh1P, and that Sqh2P is more strongly expressed in the folds of the epithelium (arrowheads) than in the non folded regions (denoted by a bracket). (E) Schematic illustration of a sagittal section of a wing disc. (Scale bars $=20 \mu \mathrm{m}$ ) 

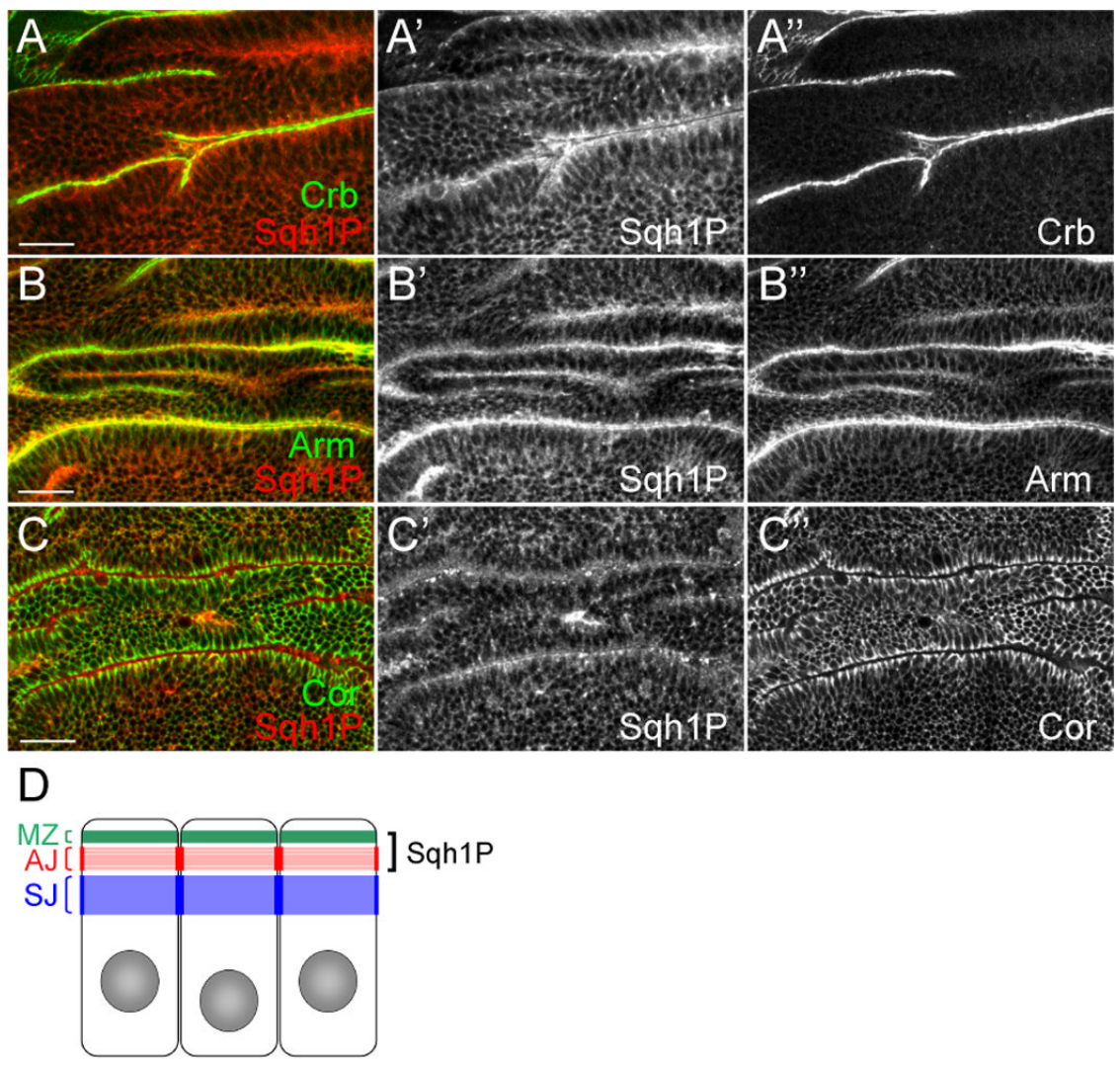

Fig. 7. Sqh1P localizes primarily to the adherens junction in wing imaginal disc cells (A-C) Confocal optical sections of $3^{\text {rd }}$ instar wing discs double labeled with Sqh1P (red) and Crb (green) (A), Sqh1P (red) and Arm (green) (B), and Sqh1P (red) and Cor (green) (C). Individual Sqh1P channels are shown in ', with the second antibody shown in ". Note the strongest colocalization is between Sqh1P and Arm, an adherens junction marker. (D) Schematic illustration indicating the primary localization for Sqh1P is in the adherens junction in imaginal disc epithelial cells. (Scale bars $=20 \mu \mathrm{m})$ 


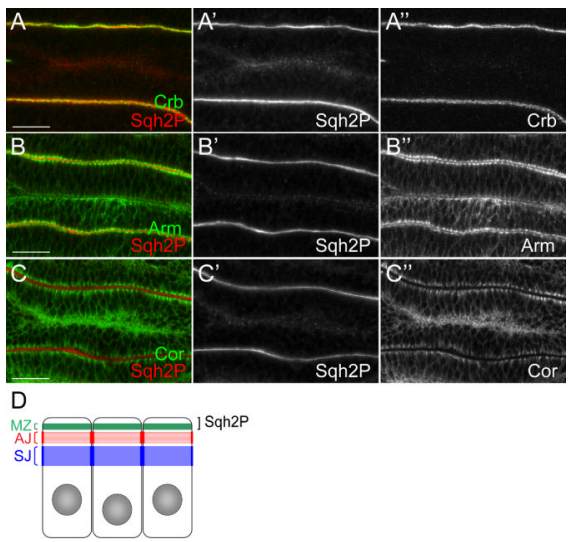

Fig. 8. Sqh2P localizes primarily to the apical surface of wing imaginal disc cells (A-C) Confocal optical sections of $3^{\text {rd }}$ instar wing discs double labeled with Sqh2P (red) and Crb (green) (A), Sqh2P (red) and Arm (green) (B), and Sqh2P (red) and Cor (green) (C). Individual Sqh2P channels are shown in ', with the second antibody shown in ". Note the strongest colocalization is between Sqh2P and Crb, a marginal zone marker, although Sqh2P is clearly even more apical than Crb. (D) Schematic illustration indicating the primary localization for Sqh2P is in the marginal zone and apical plasma membrane in imaginal disc epithelial cells. (Scale bars $=20 \mu \mathrm{m})$ 